\title{
Benefícios da estimulação perceptual corporal no esquema corporal de idosos
}

\section{Benfits of body perceptionsimulationonbooly sherearengtheddaly}

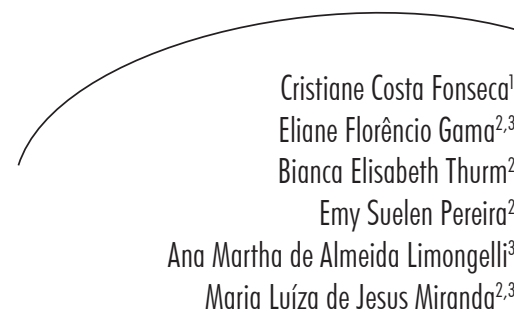

\section{Resumo}

Os avanços da ciência proporcionaram o aumento da longevidade e refletem o crescimento do contingente de idosos na sociedade atual. $\mathrm{O}$ aumento da idade cronológica traz perda progressiva da funcionalidade e altera o processo de fornecimento de informações sobre a percepção corporal, resultando na interpretação distorcida dos parâmetros corporais. Estudos comprovam que o movimento e atividade física são capazes de influenciar positivamente a percepção corporal. O objetivo desta pesquisa foi avaliar os efeitos de atividades de estimulação corporal perceptual sobre o esquema corporal dos idosos. A avaliação foi realizada através do teste de projeção de pontos, o IMP (Image Marking Procedure), que é capaz de predizer o grau de distorção entre a imagem real e percebida nos diferentes pontos do corpo. As medidas de percepção das dimensões corporais foram mensuradas antes e depois de um programa de estimulação perceptual corporal. Os resultados do estudo demonstraram que o programa de intervenção corporal pode trazer benefícios para os sujeitos participantes, mas é necessário que a estimulação tenha um foco mais particular conforme as características perceptivas de cada indivíduo.

Abstract

Advances in science have resulted in increased longevity and reflect the growing number of elderly individuals in society today. The increase in chronological age brings progressive loss of functionality, and changes the process of providing information on body awareness, resulting in the misinterpretation of body parameters. Studies have shown that movement and physical activity are able to positively influence body perception. This study aimed to evaluate the effects of perceptual body stimulation activities on the body schema of the elderly. The evaluation was performed with the IMP

Palavras-chave:

Envelhecimento. Imagem Corporal. Atividade Motora. Cinésica. Idoso. Atividade física.

\footnotetext{
Faculdade de Fisioterapia. Universidade Federal de Alfenas.Alfenas, MG, Brasil.

Programa de Pós-Graduação em Educação Física. Universidade São Judas Tadeu. São Paulo, SP, Brasil.

Programa de Pós-Graduação em Ciências do Envelhecimento. Universidade São Judas Tadeu. São Paulo, SP, Brasil.
}

Key words: Aging. Body Image. Motor Activity. Kinesics. Aged. Physical Activity. 
(Image Marking Procedure), capable of measuring the degree of distortion between the actual and perceived image in different parts of the body. Measurements of body size perception were taken before and after perceptual body stimulation programs. The study results showed that the body intervention program can bring benefits to the participants, but it is necessary that stimulation have a more specific focus, according to the perceptive characteristics of each individual.

\section{INTRODUÇÃO}

O envelhecimento humano é um fenômeno muito discutido e investigado na sociedade atual. O aumento da longevidade e a maior expectativa de vida decorrente dos avanços da ciência refletem o crescimento do contingente de idosos. ${ }^{1}$

Envelhecer implica alterações físicas e psicológicas. O aumento da idade cronológica traz alterações nas dimensões corporais. As variáveis proprioceptivas sofrem modificações: idosos apresentam diminuição de sensibilidade, alteração na coordenação motora, têm um maior tempo de resposta dos músculos efetores, perda de equilíbrio e menor destreza nos movimentos. ${ }^{2}$

A percepção das dimensões corporais nessa idade está relacionada com a integridade do sistema nervoso e do esquema corporal. Este é um aspecto neurológico que representa as relações espaciais desse indivíduo entre as partes do corpo percebidas cinestésica e proprioceptivamente. Caracterizase por uma interação neuromotora que permite perceber o próprio corpo no espaço e desenvolver as ações de forma adequada. O esquema corporal é dinâmico, ou seja, alterações posturais, objetos, espaço próximo ao alcance dos segmentos se qualquer situação que participe do movimento consciente do corpo é somada ao modelo corporal, tornando-se parte desse esquema. ${ }^{3-5}$

O desgaste natural do organismo, característico do idoso, provoca perda na multiplicidade do sistema sensorial e altera o processo de fornecimento de informações sobre a percepção corporal. ${ }^{6} \mathrm{O}$ envelhecimento traz também impactos emocionais e uma interpretação distorcida dos parâmetros corporais. ${ }^{7} \mathrm{O}$ insucesso na tentativa de reverter as mudanças características do envelhecimento, a não-aceitação do processo de declínio e a exigência social por um ideal físico difícil de ser alcançado geram insatisfação com a aparência. Assim, estudar formas de minimizar a intensidade das alterações fisiológica e o desenvolvimento de mecanismos que melhorem a satisfação e adaptação do idoso a sua condição e a criação de estratégias que promovam qualidade no envelhecimento são imprescindíveis. ${ }^{8,9}$

Estudos comprovam que o movimento e atividade física são capazes de influenciar a percepção corporal. ${ }^{6,10,11}$ Trabalhar a percepção do próprio corpo e desenvolver o sentimento de aceitação das alterações pertinentes a idade são fundamentais para manter a auto-estima, bemestar físico e emocional nessa fase da vida. ${ }^{11,12}$

Esses dados trazem a necessidade de refletir sobre a necessidade de analisar de forma científica a intensidade com que as alterações do envelhecimento afetam a percepção que o indivíduo tem do próprio corpo, suas implicações na sua satisfação física e quais efeitos o exercício tem em retardar e reverter esse processo. Assim, o objetivo deste estudo foi avaliar os efeitos de atividades de estimulação corporal perceptual sobre o esquema corporal dos idosos participantes de um projeto de intervenção em educação e saúde de idosos, denominado Projeto Sênior para Vida Ativa.

\section{MATERIAIS E MÉTODOS}

Sujeitos

Participaram desse estudo 21 idosos de ambos os sexos, integrantes do Projeto Sênior 
para a Vida Ativa da Universidade São Judas Tadeu, São Paulo. Os critérios para inclusão no estudo foram: idade superior a 60 anos, possuir qualquer nível de escolaridade e estar classificado nos níveis três ou quatro de status funcional, conforme proposto por Spirduso, ${ }^{2}$ ou seja, ser independente e inativo (nível 3) ou já ativo (nível 4), ter frequência superior a $85 \%$ nas aulas ministradas nos projetos para os praticantes e portar autorização médica para a prática de atividades físicas. Foram excluídos da pesquisa indivíduos que apresentaram qualquer dificuldade de compreensão que prejudicasse o entendimento das avaliações realizadas.

Todos os participantes foram informados dos objetivos e procedimentos do estudo, bem como da possibilidade reduzida de riscos na sua participação. Ressalta-se que aqueles que concordaram em participar assinaram o termo de consentimento livre e esclarecido, Protocolo $n^{\circ}$ 083/09 e Sisnep no 67.0.219.000-9, segundo as normas do Comitê de Ética em Pesquisa da Universidade São Judas Tadeu, e estavam livres para interromper a pesquisa no momento em que quisessem.

\section{Coleta de dados}

A coleta de dados foi realizada em 21 idosos dos 32 participantes do Projeto Sênior para Vida Ativa. Foram excluídos do estudo os idosos que não participaram das seis intervenções de estimulação perceptual. Em relação às características sociodemográficas, todos são aposentados, de classe média e residem próximo à Universidade São Judas Tadeu. A grande maioria é do sexo feminino e apresenta uma média de 71,24 $\pm 7,74$ anos. Dezoito dos 21 participantes informaram que realizam outras atividades físicas além das oferecidas no projeto. As atividades complementares praticadas são a caminhada, alongamento, tai chi chuan e hidroginástica, e são realizadas em média duas vezes por semana. O Projeto Sênior para Vida Ativa tem caráter educacional e está organizado em torno de uma proposta de educação física cujo objetivo é difundir conhecimentos sobre exercícios e proporcionar a prática regular de atividade física. ${ }^{13}$

No início das atividades do projeto, os voluntários participaram de sessões de estimulação perceptual compostas por movimentos que forneciam estímulos proprioceptivos e exteroceptivos, envolvendo atividades como rolar no chão, exercícios com bola, transferência de peso, movimentos de alcance e comparação de sensações entre antímeros do corpo depois de um dado estímulo. É fundamental observar o componente principal dessa intervenção, que a diferenciava de uma aula de alongamento ou ginástica habitual: o foco da atenção na parte do corpo que estava sendo trabalhada e o destaque dado à sensação percebida depois de cada movimento. $^{14}$

A intervenção foi composta por seis sessões com 50 minutos de duração cada. As análises foram feitas antes da primeira intervenção e logo após a última.

\section{Instrumentos de avaliação}

A avaliação do esquema corporal é realizada por meio do teste de projeção de pontos, o IMP (Image Marking Procedure), ${ }^{15,16}$ capaz de predizer o grau de distorção entre a imagem real e percebida nos diferentes pontos do corpo (alto da cabeça, ombros, cintura e quadril).

Os sujeitos são marcados com uma etiqueta nas seguintes regiões corporais: articulações acromioclavicular direita e esquerda, curvas da cintura direita e esquerda, trocânteres maiores do fêmur direito e esquerdo. Esse procedimento tem como objetivo garantir que sempre sejam tocados os mesmos pontos em todos os testes.

Para avaliar o esquema corporal por meio do IMP, os sujeitos devem permanecer em posição ortostática diante de uma parede branca. A distância do sujeito até a parede é determinada pelo comprimento do membro superior do sujeito semiflexionado, de forma que sua mão atinja a parede. O teste é realizado com o sujeito de olhos vendados e com instrução verbal para 
imaginar que a parede é um espelho e que sua imagem está sendo refletida nele. Em seguida, o sujeito é tocado em pontos marcados com fita adesiva e é orientado a apontar na parede com o membro do respectivo lado que está sendo tocado a projeção desse ponto. ${ }^{15,16}$

O primeiro ponto anatômico avaliado é o alto da cabeça. Nesse momento, o indivíduo deve fazer uma apneia inspiratória. São realizadas três medidas consecutivas para cada ponto sem que o examinado veja as marcações anteriores. Em seguida, o pesquisador posiciona o sujeito próximo à parede e marca a posição real dos pontos tocados. Cada tentativa é representada com uma etiqueta colorida (vermelha, preta e verde) colada na parede, e medida real, por sua vez, é representada por etiquetas azuis. A imagem formada é então fotografada para posterior análise das medidas. Para evitar erros de marcação dos pontos reais, o pesquisador estabiliza a região cervical com o apoio da mão para impedir o balanço postura. São consideradas a medida da estatura e as distâncias na horizontal dos pontos marcados que representam a largura corporal. ${ }^{11}$ Após a marcação dos pontos, é aplicado o índice de percepção corporal (IPC), que consiste em calcular a fórmula: tamanho percebido dividido pelo tamanho real multiplicado por $100 .^{11,15,17}$

O presente trabalho adota como critério de classificação do IPC a mesma classificação descrita por Pereira ${ }^{17}$ em seu estudo, que analisou 146 indivíduos de 11 a 75 anos. Indivíduos que apresentem IPC inferior a $102,28 \%$ são considerados hipoesquemáticos, valores maiores ou iguais a $102,28 \%$ e menores ou iguais a $123,58 \%$ são classificados como esquema corporal adequado e valores superiores a 123,58\% são considerados hiperesquemáticos. ${ }^{17}$

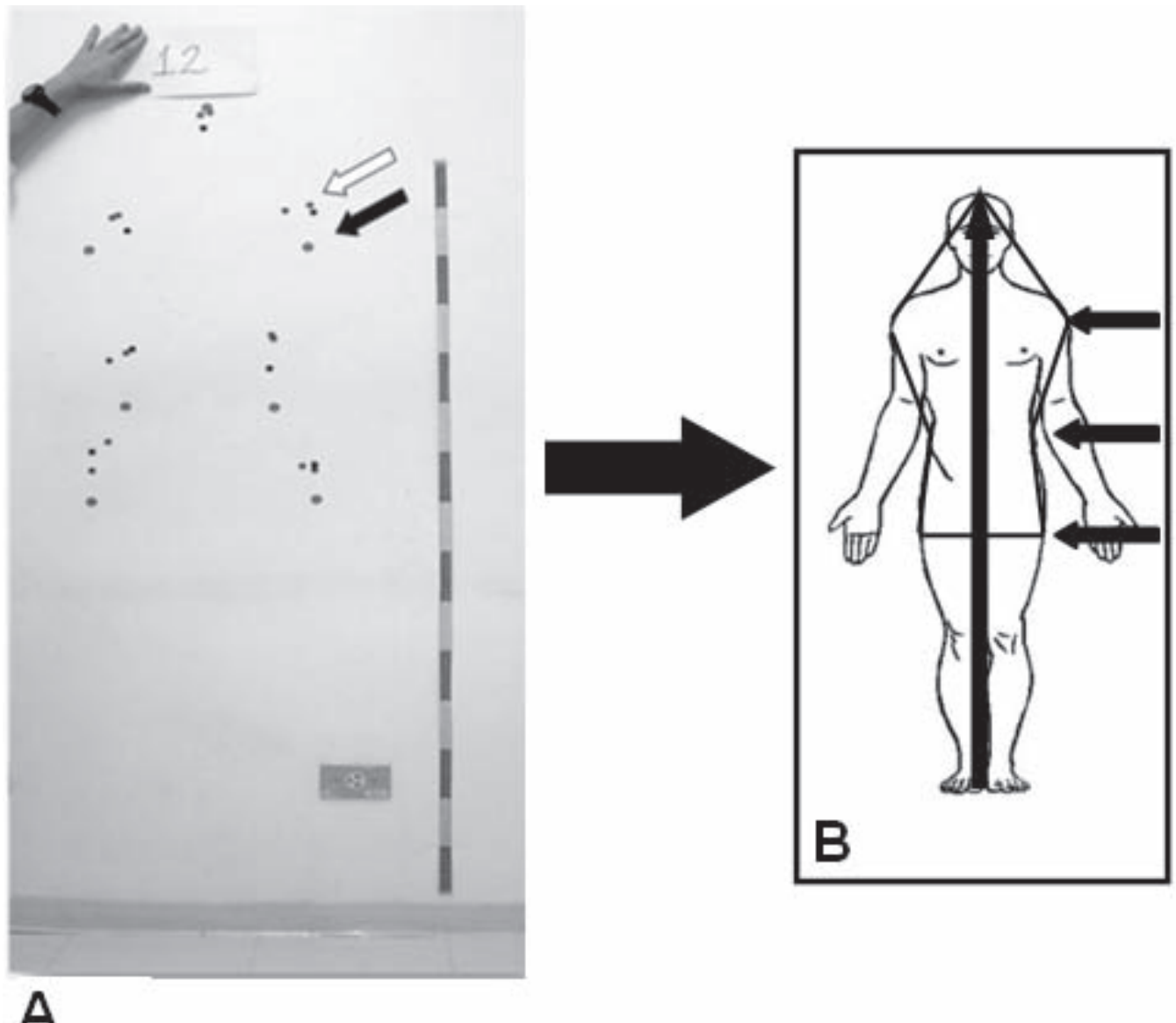

Figura 1. A: Teste de Marcação do Esquema Corporal B: Esquema representativo das dimensões corporais avaliadas 
Análise de dados

Foram avaliados os IPCs dos seguintes segmentos: altura de cabeça, largura do ombro, da cintura e do quadril. A análise quantitativa dos resultados do esquema corporal, o IPC, foi feita com o uso do software estatístico SPSS (Statistical Package for Social Science), versão 12.0, e o nível de significância adotado foi de $5 \%$. Os resultados foram apresentados com média e desvio padrão.

Para analisar o IPC geral dos participantes, foi utilizado o teste $t$ Student para verificar as múltiplas comparações entre os grupos no momento inicial e depois da intervenção perceptual. Para análise estatística de cada segmento separadamente e comparação entre essas medidas entre si nos diferentes momentos (antes e depois da estimulação perceptual), foi utilizado o teste ANOVA com medidas repetidas e as diferenças entre os pares foram avaliadas com o teste de Bonferroni.

A análise qualitativa das imagens digitalizadas da mensuração do esquema corporal foi feita por meio da observação dos desenhos formados a partir da união dos pontos projetados. Para isso, foi feita uma comparação visual entre as distorções e similaridades da dimensão real (linha contínua) e percebida (linha pontilhada). Comparou-se o desenho real e percebido apresentado nas primeiras aulas com o desenho traçado das medidas real e percebida no final das aulas. Caso a linha vermelha estivesse mais próxima da preta, isso foi considerado sinal indicativo de melhora na percepção corporal. Foi observada também a presença ou não de assimetrias e/ou desvios da linha mediana.
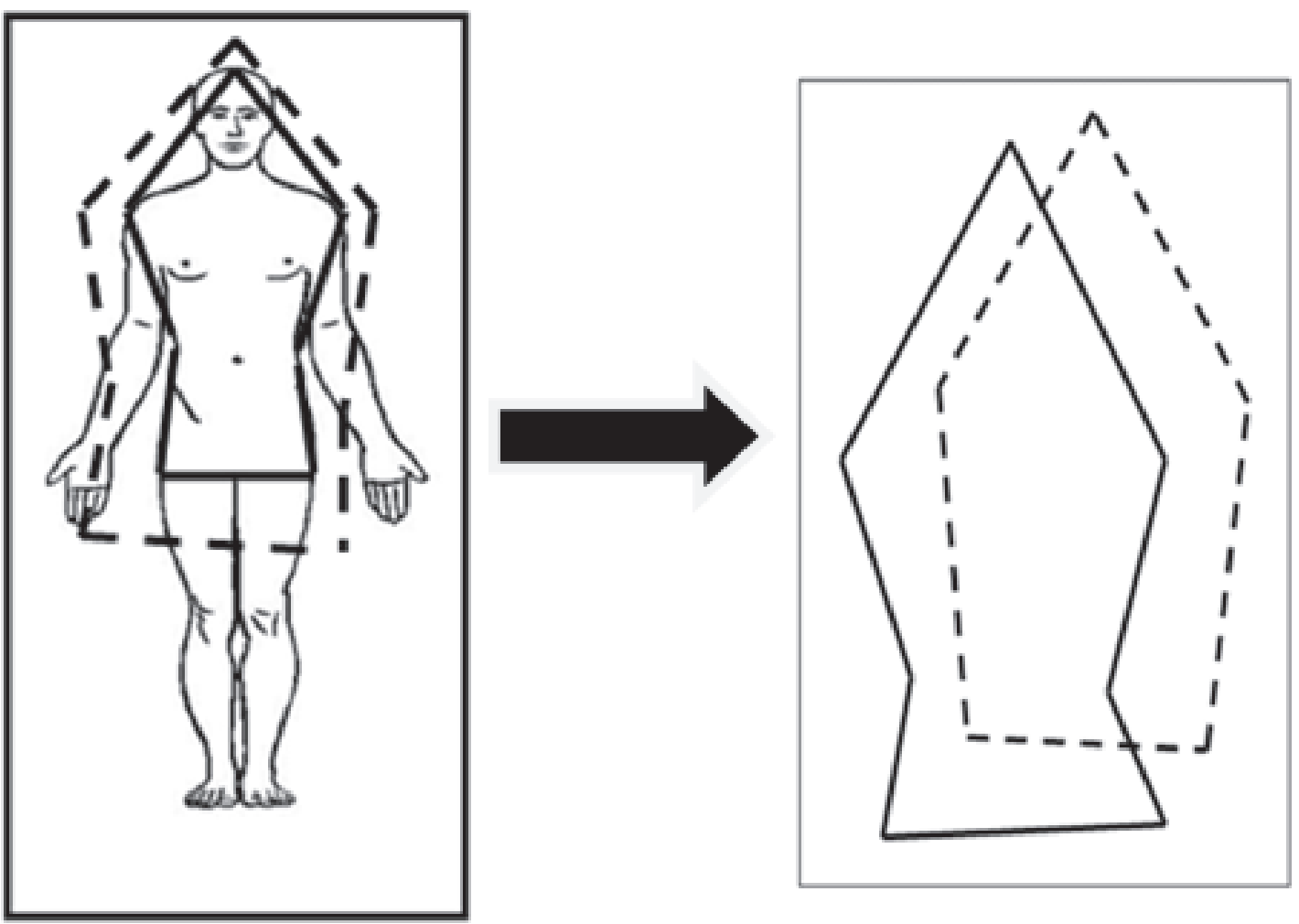

Figura 2. Desenho obtido ao final do procedimento de marcação do esquema corporal. Dimensão corporal percebida (linha pontilhada) e dimensão corporal real (linha contínua). 


\section{RESULTADOS}

Análise Quantitativa

Quando foram comparadas as médias do IPC geral dos sujeitos antes $(111,8 \pm 27,4 \%)$ e depois $(108,97 \pm 14,86 \%)$ da estimulação perceptual e aplicada a análise estatística $t$ Student, observouse que os indivíduos não apresentaram diferença estatisticamente significante $(p>0,05)$. O índice geral de percepção corporal mantevese o mesmo depois da intervenção. Conforme o critério adotado nesta pesquisa, seguindo a classificação descrita por Pereira, ${ }^{17}$ percebese que os idosos se apresentavam com um esquema corporal adequado tanto antes quanto depois da intervenção.

No entanto, quando são comparados os segmentos corporais (estatura, largura de ombros, cintura e quadril) entre si nos momentos antes e depois da intervenção, percebe-se que os sujeitos apresentaram algumas modificações. No início, os sujeitos apresentavam diferença estatisticamente significante entre O IPC da estatura $(97,79 \pm 4,59 \%) \times$ IPC da largura ombros $(112,48 \pm 23,61 \%)$ e IPC da estatura $(97,79 \pm$ $4,59 \%)$ x IPC da largura cintura $(114,81 \pm 29,8 \%)$, sendo os ombros e cintura superestimados.

Tabela 1 - Comparação dos valores da média e desvio padrão do IPC $(\%)$ dos segmentos corporais comparados entre si, antes do programa de estimulação perceptual. São Paulo, SP, 2010

\begin{tabular}{ccc}
\hline SEGMENTOS (Antes) & $t$ & Sig \\
\hline $\operatorname{ALTCAB}(97,79 \pm 4,59 \%)$ vs LARO $(112,48 \pm 23,61 \%)$ & 2,99 & $\mathrm{p}<0,05^{*}$ \\
$\operatorname{ALTCAB}(97,79 \pm 4,59 \%)$ vs LARC $(114,81 \pm 29,8 \%)$ & 3,46 & $\mathrm{p}<0,01 * *$ \\
$\operatorname{ALTCAB}(97,79 \pm 4,59 \%)$ vs LART $(102,65 \pm 21,93 \%)$ & 0,98 & $\mathrm{p}>0,05$ \\
$\operatorname{LARO}(112,48 \pm 23,61 \%)$ vs LARC $(114,81 \pm 29,8 \%)$ & 0,47 & $\mathrm{p}>0,05$ \\
$\operatorname{LARO}(112,48 \pm 23,61 \%)$ vs LART $(102,65 \pm 21,93 \%)$ & 2,00 & $\mathrm{p}>0,05$ \\
$\operatorname{LARC}(114,81 \pm 29,8 \%)$ vs LART $(102,65 \pm 21,93 \%)$ & & $\mathrm{p}>0,05$ \\
\hline
\end{tabular}


Quando foi analisada a percepção da dimensão corporal depois da intervenção, observou-se diferença estatisticamente significante entre IPC da estatura $(99,76 \pm 3,26 \%)$ x IPC da largura cintura $(116,58 \pm 24,86 \%)$, ainda com a dimensão da cintura superestimada, porém para largura de ombro houve alteração. Os sujeitos depois da estimulação perceptual não mais apresentavam diferença estatisticamente significante entre $\mathrm{O}$ IPC da estatura em relação ao IPC do ombro.

Tabela 2 - Comparação dos valores da média e desvio padrão do IPC(\%) dos segmentos corporais comparados entre si, depois do programa de estimulação perceptual. São Paulo, SP, 2010

\begin{tabular}{ccc}
\hline SEGMENTOS (Depois) & $t$ & Sig \\
\hline $\operatorname{ALTCAB}(99,76 \pm 3,26 \%)$ vs LARO $(112,42 \pm 25,5 \%)$ & 2,45 & $\mathrm{p}>0,05$ \\
$\operatorname{ALTCAB}(99,76 \pm 3,26 \%)$ vs LARC $(114,81 \pm 29,8 \%)$ & 3,26 & $\mathrm{p}<0,05 *$ \\
$\operatorname{ALTCAB}(99,76 \pm 3,26 \%)$ vs LART $(107,15 \pm 20,97 \%)$ & 1,43 & $\mathrm{p}>0,05$ \\
$\operatorname{LARO}(112,42 \pm 25,5 \%)$ vs LARC $(114,81 \pm 29,8 \%)$ & 0,80 & $\mathrm{p}>0,05$ \\
$\operatorname{LARO}(112,42 \pm 25,5 \%)$ vs LART $(107,15 \pm 20,97 \%)$ & 1,02 & $\mathrm{p}>0,05$ \\
$\operatorname{LARC}(114,81 \pm 29,8 \%)$ vs LART $(107,15 \pm 20,97 \%)$ & & $\mathrm{p}>0,05$ \\
\hline
\end{tabular}

ALTCAB (altura da cabeça), LARO (largura do ombro), LARC (largura da cintura), LART (largura do trocânter).

\section{Análise qualitativa}

$\mathrm{Na}$ análise qualitativa dos contornos da silhueta real e percebida, antes e depois da estimulação perceptual, as figuras representam a imagem percebida (linha pontilhada) e real (linha contínua) dos participantes. Quando são comparadas as imagens digitalizadas das silhuetas dos idosos antes e depois da prática de estimulação perceptual, observa-se que na maioria dos casos houve maior aproximação da silhueta percebida em relação a silhueta real do indivíduo avaliado.
Ao serem classificados em valores percentuais, 61\% (indivíduos 1,4,5,6,9,11,12,13, $14,15,16,18$ e 21) dos idosos apresentaram maior centralização da imagem, bem como melhor dimensionamento da silhueta; 28\% (indivíduos 7,8,10,17,19 e 20) dos participantes não apresentaram variação entre a silhueta real e percebida depois da estimulação e somente 9,5\% (indivíduos 2 e 3 ) apresentaram piora das imagens após a prática. 

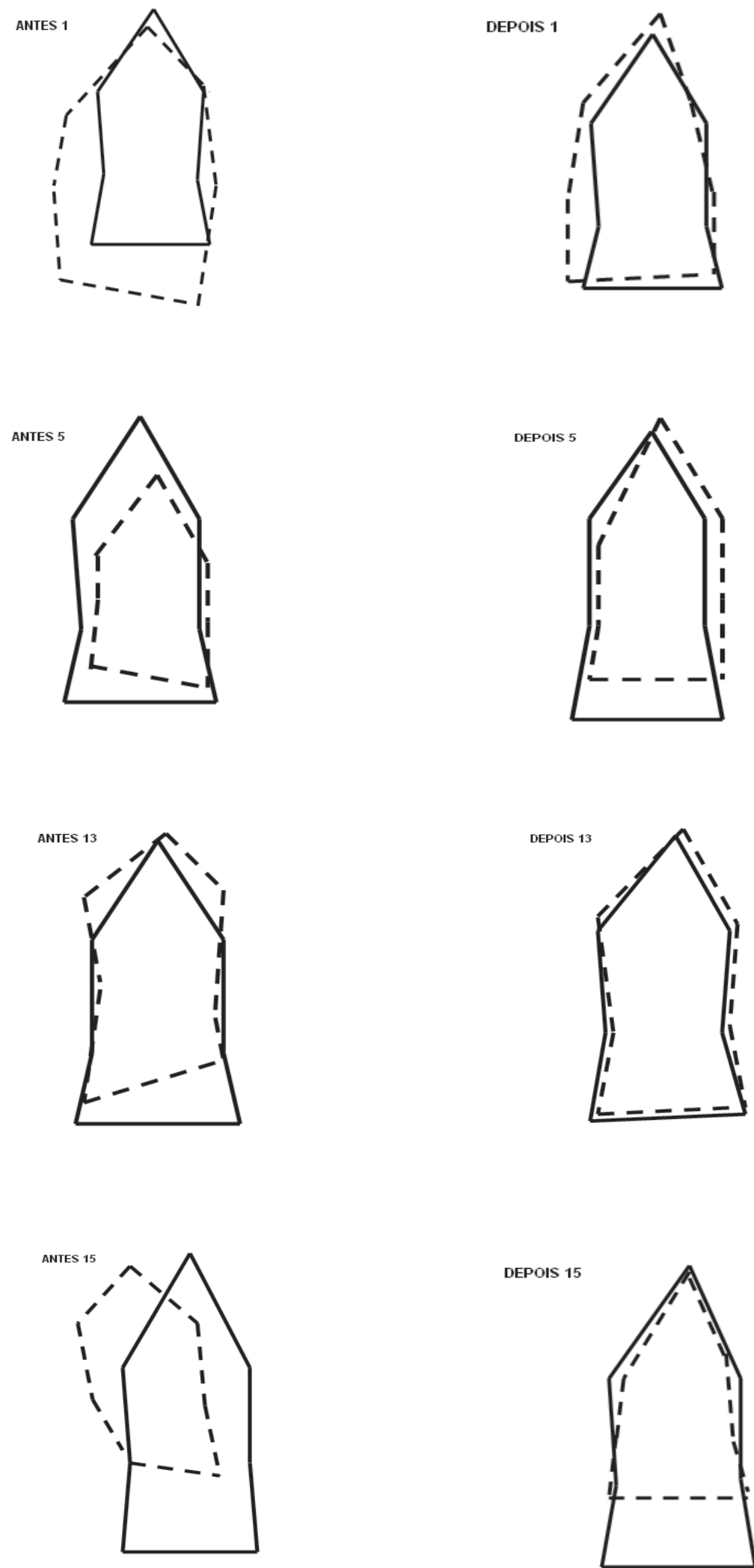

Figura 3. Comparação do desenho das silhuetas apontadas pelos sujeitos antes e depois do programa de intervenção. 


\section{DISCUSSÃO}

$\mathrm{Na}$ análise quantitativa dos resultados do IPC geral dos participantes antes e depois da estimulação perceptual, percebe-se que os sujeitos não apresentaram diferença estatisticamente significante entre os valores. No entanto, numa observação mais detalhada é possível perceber que antes da estimulação os idosos já apresentavam percepção corporal satisfatória. $\mathrm{O}$ estudo de Pereira et al. ${ }^{17}$ cujo objetivo foi delinear um padrão médio do IPC, apontou que valores de normalidade para o esquema corporal estão entre $102,28 \%$ a $123,58 \%$. Quando se adota essa mesma classificação para o presente estudo, verifica-se que os indivíduos na primeira avaliação já estavam dentro do intervalo de normalidade, ou seja, apresentavam boa percepção corporal.

A explicação para essa acurácia prévia na percepção corporal dos idosos pode estar no fato de que esses já praticavam atividade física antes de ingressarem no programa. Ao analisar o histórico de atividade física dos indivíduos (18 dos 21 sujeitos avaliados forneceram essa informação), observase que $86 \%$ deles já eram ativos antes realizarem as intervenções. Ao analisar os exercícios realizados antes da inclusão no estudo, o mais praticado foi caminhada $-72 \%$ dos indivíduos praticavam essa atividade pelo menos duas vezes por semana. Seguiram-se musculação (22\%), ginástica $(22 \%)$ hidroginástica $(17 \%)$ e alongamento (17\%), sendo que $81 \%$ dos sujeitos realizavam mais de uma modalidade durante a semana.

A prática regular de atividade física pode ter contribuído para retardar o declínio físico natural da idade e, consequentemente, ter influenciado na percepção corporal apresentada por esses idosos antes da intervenção. A influência do exercício na percepção corporal foi comentada na literatura por Frederic, ${ }^{18}$ que descreveu modificações positivas estatisticamente significantes na imagem corporal de idosos depois que esses frequentaram um programa de atividade física. Matsuo et al. ${ }^{19}$ concordam com essa afirmativa, destacando que a atividade física é essencial para ampliar o conhecimento do corpo, sobretudo nos idosos, e dentre todos os benefícios está o de reconhecer os limites e especificidade do corpo envelhecido. Como esses idosos já praticavam atividade física antes do programa de estimulação perceptual, os efeitos benéficos significantes do exercício na saúde e percepção já estavam expressos no corpo.

Um aspecto a ser levado em consideração na elaboração, planejamento e aplicação são as características do programa de intervenção sobre a percepção corporal. Uma limitação deste trabalho é que os benefícios do programa de estimulação perceptual poderiam ser mais efetivos se elaborados de modo a atender às necessidades individuais. Neste estudo, o protocolo foi aplicado de modo a estimular a percepção corporal geral, não levando em consideração as necessidades específicas. Por exemplo, seria mais adequado para aqueles indivíduos que tivessem maior alteração na percepção do quadril poderiam ter sido enfatizados mais exercícios que estimulassem essa região, ou idosos que apresentassem maior distorção na percepção do ombro poderiam realizar mais atividades com enfoque nos membros superiores, com o intuito de obter mais benefício do programa de estimulação perceptual.

Benedetti et al., ${ }^{20}$ em pesquisa sobre exercícios físicos e autoimagem de idosos na qual compararam idosos asilados sedentários com idosos que participaram de um programa de atividade física, comprovaram que a prática de atividade física promoveu a melhora estatística da autoimagem e autoestima. E Fonseca, ${ }^{11} \mathrm{em}$ estudo sobre a influência da dança de salão na percepção corporal, demonstrou que a atividade traz benefícios significantes para o esquema corporal. O trabalho avaliou a influência da dança de salão no esquema corporal e imagem corporal de 15 indivíduos praticantes, demonstrando que a prática por três meses foi capaz de melhorar a percepção corporal, apresentando significância estatística tanto no aspecto neuromotor quanto em relação a satisfação corporal. Ressalta-se que, 
dos indivíduos analisados, oito tinham entre 45 a 60 anos. Essa faixa etária é denominada envelhescente, ou seja, fase de transição da maturidade para velhice. Confirmou-se que a dança não só teve efeitos positivos sobre a percepção corporal, como os benefícios foram detectados em indivíduos já no processo de declínio físico. ${ }^{11,21}$

Vale destacar que, nesses estudos, o públicoalvo apresentava distorções da percepção corporal antes de ingressar no programa e foi submetido a algum tipo de atividade não-individualizada. Diferentemente da população deste trabalho, os idosos já apresentavam boa percepção corporal e, provavelmente necessitariam de uma análise e intervenção individualizada. Essa percepção previamente acurada antes do início da pesquisa foi apresentada pelos participantes também é mais uma limitação do presente estudo.

A importância de um trabalho individual é ressaltada quando comparamos os desenhos das figuras da dimensão corporal na análise qualitativa dos contornos da silhueta real e percebida, antes e depois da estimulação perceptual. Quando são observados os indivíduos 13 e 15, existe clara distorção na percepção da região do quadril. Para esses sujeitos, seria ideal uma maior abordagem para a região pélvica. Em contrapartida, quando o sujeito 5 é analisado, percebe-se que apresenta maior distorção na região do ombro. Certamente esse indivíduo seria mais beneficiado com atividades que estimulassem a região superior. Já quando se presta atenção no sujeito 1 , observa-se que sua distorção é mais localizada na área da cintura - para esse sujeito, diferentemente dos outros, o enfoque deveria ser na área da cintura.

Apesar de os resultados do IPC geral não terem sido expressos numericamente na análise estatística, quando são analisados os resultados de forma qualitativa, através da comparação das figuras das silhuetas formadas com a união dos pontos das imagens, percebe-se que a maior parte dos sujeitos depois da estimulação perceptual promoveu melhor ajustamento do contorno corporal. A variação da percepção corporal pode limitar o estudo, devido às múltiplas variações possíveis entre os indivíduos. Assim, a análise das imagens é uma forma de avaliação mais específica, pois além de observar cada indivíduo caso a caso, permite detectar variações de centralização e simetria, ao contrário do teste numérico, que além de reduzir todos indivíduos em um único padrão, é específico para avaliar somente alterações na largura dos segmentos.

Os resultados qualitativos demonstram que as atividades propostas promoveram maior simetria e centralização do contorno corporal. Sendo assim, observa-se que o programa de estimulação perceptual trouxe benefícios para a percepção corporal dos idosos participantes do estudo. Pesquisas como a de Cecchini et al. ${ }^{22}$ descreveram a influência de um programa de vivências corporais na ampliação da percepção corporal de estudantes e demonstraram que, através da estimulação, os indivíduos puderam redimensionar sua compreensão de corpo, observar mais atentamente suas sensações e que as técnicas vivenciadas trouxeram melhora para percepção corporal desses indivíduos. É importante salientar que as atividades utilizadas estimulavam o desenvolvimento da noção de corpo e eram semelhantes à proposta de atividades adotadas na presente pesquisa, para estimulação perceptual.

O estudo de Fonseca $^{11}$ corrobora o pressuposto deste estudo, afirmando que a dança de salão, uma forma de estimulação corporal rítmica e que exige atenção, é capaz de trazer benefícios para percepção corporal. No entanto, vale destacar a escassez de trabalhos que avaliem os efeitos da intervenção sobre a percepção corporal. Existem muitas referências sobre percepção corporal, mas a maior parte se preocupa em analisar aspectos do fenômeno em si. Poucos são os estudos que propõem recursos de intervenção e formas de desenvolvimento da percepção corporal. São necessários mais estudos que proponham formas positivas de se trabalhar o redimensionamento corporal. 


\section{CONCLUSÃO}

Através desta pesquisa, podemos concluir o pressuposto de que a estimulação perceptual é capaz de provocar modificações positivas no esquema corporal e promover maior

\section{REFERÊNCIAS}

1. IBGE. Brasil em síntese: 2000. [acesso em 05 nov. 2009]. Rio de Janeiro IBGE, 2006. Disponível em: URL:< http://www.ibge.gov.br/brasil_em_sintese/ default.htm $>$

2. Spirduso W. Dimensões físicas do envelhecimento. 2ed. Barueri: Manole, 2005.

3. Barros DD. Imagem corporal: A descoberta de si mesmo. História, Ciência, Saúde. 2005; 12(2): 547-54.

4. Maravita A, Irki A. Tools for The Body (Schema). Trends Cogn Sci 2004 Feb.; 8(2):79-86.

5. Medina J, Coslett BH. From maps to form to space: touch and the body schema. Neuropsychologia. 2010 feb.; 48(3): 645-54.

6. Benvenuto MC. Uma avaliação acerca do perfil perceptivo motor de mulheres idosas submetidas a uma tarefa rítmica-sonora. Ulbra e Movimento Revista de Educação Física. 2010; 1(1):1-11.

7. Guerra ACLC, Caldas, CP. Dificuldades e recompensas no processo de envelhecimento: a percepção do sujeito idoso. Cien Saude Colet 2010 set.; 15(6): 2931-8.

8. Varejão RV, Dantas EHM, Matsudo SMM. Comparação dos efeitos do alongamento e do flexionamento, ambos passivos sobre os níveis de flexibilidade, capacidade funcional e qualidade de vida do idoso. Revista brasileira de ciência \& movimento. 2007; 15(2): 87-95.

9. Matsudo SM, Matsudo VKR. Impacto do envelhecimento nas variáveis antropométricas, neuromotoras e metabólicas da aptidão física. Revista brasileira de ciência \& movimento 2000; 8(4): 21-32.

10. Turtelli LS. Relações entre Imagem Corporal e Qualidade de Movimento: Uma Reflexão A Partir de uma Pesquisa Bibliográfica. [dissertação] Campinas, SP: UNICAMP, Programa de Mestrado em Educação Física; 2000.

11. Fonseca CC. Análise do Esquema corporal, imagem corporal na dança de salão e seus aspectos motivacionais. [dissertação]. São Paulo, autoaceitação. No entanto, conforme discutido no presente estudo, para futuras intervenções seria importante que fossem desenvolvidos programas individuais específicos enfatizando áreas de maior distorção, para que os benefícios obtidos possam ser ainda mais significativos.
SP: Universidade São Judas Tadeu, Programa de Mestrado em Educação Física; 2008.

12. Tribess S. Percepção da imagem corporal e fatores relacionados à saúde em idosas. [dissertação]. Florianópolis, SC: Universidade Federal de Santa Catarina, Programa de Mestrado em Educação Física, 2006.

13. Miranda MLJ, Velardi M. A proposta de educação física do Projeto Sênior para a Vida Ativa da USJT: contribuições para uma velhice bem sucedida. $\mathrm{V}$ Seminário Internacional sobre Atividade Física para a Terceira Idade; 2002 nov; São Paulo. São Paulo: USP/EEF; 2002.

14. Thurm BE, Cangelli Filho R, Gama EF. Estimulação corporal perceptual: Uma abordagem terapêutica nos transtornos alimentares. I Simpósio Internacional de Imagem Corporal e I Congresso Brasileiro de Imagem Corporal; 2010 ago; São Paulo. Campinas: UNICAMP/FEF; 2010.

15. Thurm BE. Efeitos da Dor Crônica em Atletas de Alto Rendimento em Relação ao Esquema Corporal, Agilidade Psicomotora e Estados de Humor. [dissertação]. São Paulo, SP: Universidade São Judas Tadeu, Programa de Mestrado em Educação Física, 2007.

16. Askevold F. Measuring Body Image: Preliminary Report in a New Method Psychother Psychosom. 1975; 26(1):71-7.

17. Pereira ES, Miranda MLJ, Velardi M, Segheto W, Neto AR, Dantas DB, Segheto KJ, Thurm BE, Gama, EF. Comportamento do Esquema Corporal do Adolescente ao Idoso. Congresso Internacional de Envelhecimento Humano; 2010 abr; Rio Grande do Sul. Passo Fundo: PUC/RS; 2010.

18. Frederici ES. Imagem corporal de idosos praticantes de um programa de atividade física. [dissertação]. São Paulo, SP: Universidade de São Paulo, Programa de Mestrado em Educação Física e Esportes; 2004.

19. Matsuo RF, Velardi M. Brandão MRF Miranda MLJ Imagem corporal de idosas e atividade física. Revista Mackenzie de Educação Física e Esporte 2007; 6(1):37-43. 
20. Benedetti TB, Petroski EL. Gonçalves LT.

Exercícios físicos, auto-imagem e auto-estima em idosos asilados. Revista Brasileira de Cineantropometria e Desenvolvimento Humano 2003; 5(2):69-74.

21. Nogueira R, Fonseca CC, Gama EF. A influência da dança de salão na percepção corporal de indivíduos envelhescentes. I Simpósio Internacional de Imagem Corporal e I Congresso Brasileiro de Imagem Corporal; 2010 ago; São Paulo. Campinas: UNICAMP/FEF; 2010.

22. Cecchini LML, Brun MCS, Borges CK, Marques MG. A influência das vivências corporais na ampliação da percepção corporal em acadêmicos de fisioterapia: edfesportes [Internet]. 2008. [acesso em 2010 fev 03]; 10(83). Disponível em: URL:<http:// www.efdesportes.com/

Recebido: 08/7/2011

Revisado: 28/11/2011

Aprovado: 10/12/2011 